\title{
A Finite-state Machine for Accommodating Unexpected Large Ground Height Variations in Bipedal Robot Walking
}

\author{
Hae-Won Park, Alireza Ramezani, and J.W. Grizzle
}

\begin{abstract}
This paper presents a feedback controller that allows MABEL, a kneed planar bipedal robot with $1 \mathrm{~m}$-long legs, to accommodate terrain that presents large unexpected increases and decreases in height. The robot is provided information on neither where the change in terrain height occurs, nor by how much. A finite-state machine is designed that manages transitions among controllers for flat-ground walking, steppingup and down, and a trip reflex. If the robot completes a step, the depth of a step-down or height of a step-up can be immediately estimated at impact from the lengths of the legs and the angles of the robot's joints. The change in height can be used to invoke a proper control response. On the other hand, if the swing leg impacts an obstacle during a step, or has a premature impact with the ground, a trip reflex is triggered on the basis of specially designed contact switches on the robot's shins, contact switches on the end of each leg, and the current configuration of the robot. The design of each control mode and the transition conditions among them are presented. The paper concludes with experimental results of MABEL (blindly) accommodating various types of platforms, including ascent of a $12.5 \mathrm{~cm}$ high platform, stepping-off an $18.5 \mathrm{~cm}$ high platform, and walking over a platform with multiple ascending and descending steps.
\end{abstract}

\section{INTRODUCTION}

Bipedal locomotion has attracted attention for its potential ability, superior when compared to wheeled locomotion, to overcome rough terrain or environments with discontinuous supports. Existing bipedal robots, however, can only deal with small unknown variations in ground height. Ground height variations exceeding a few centimeters must be known a priori and require carefully planned maneuvers to overcome them.

Two major avenues of research are currently being pursued to quantify and improve the ability of a bipedal machine to walk over uneven terrain. A stochastic model of ground variation is being investigated in [1], [2], [3] for low-dimensional dynamical systems such as the rimless wheel and the compass bipedal walker. The mean first-passage time to the fallen absorbing state is used to assess the robustness of a gait. This metric captures the expected time that a robot can walk before falling down, measured in units of number of steps. Numerical dynamic programming is applied to a discretized representation of the dynamics to maximize the mean firstpassage time. In [4], [5], [6], the gait sensitivity norm, defined as the variance of the system's output (taken as step duration) to unit white noise input (taken as change in ground height), is introduced to quantify the ability of a bipedal robot to

H.-W. Park and A. Ramezani are with the Mechanical Engineering Department, University of Michigan, Ann Arbor, MI, 48109-2125, USA, parkhweumich.edu

J. W. Grizzle is with the Control Systems Laboratory, Electrical Engineering and Computer Science Department, University of Michigan, Ann Arbor, MI 48109-2122, USA.

This work is supported in part by NSF grant ECS-909300 and in part by DARPA Contract W91CRB-11-1-0002.

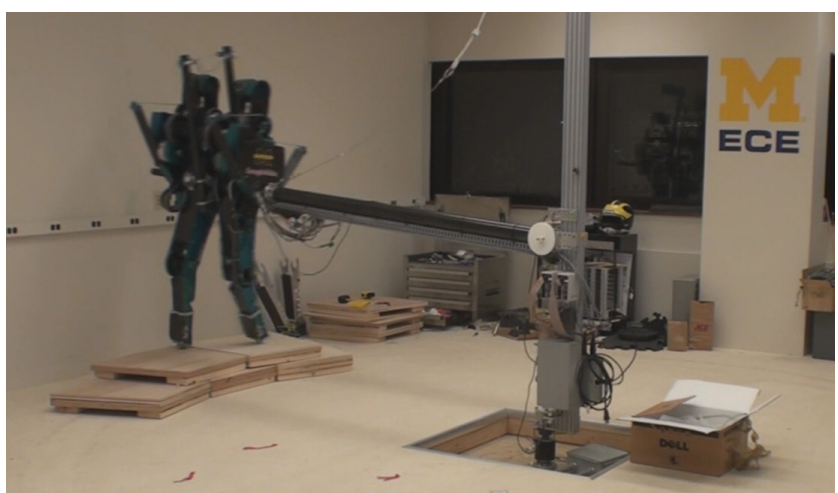

Fig. 1: MABEL, a planar testbed for bipedal locomotion, is shown traversing a platform with $10.5 \mathrm{~cm}$ and $8 \mathrm{~cm}$ steps.

handle disturbances. Particular attention is given to a "stepdown test", where the ground profile consists of a flat section, followed by an abrupt decrease in height, followed again by a flat section of ground. These references use the gait sensitivity norm to assess the improvement in disturbance rejection when swing-leg retraction speed at the end of the step is varied [5].

Along with the search for the best quantity to measure the robustness of bipedal walking to ground variation, several control design approaches for walking over uneven ground have been proposed in the literature. The work presented in [7], [8] begins with the computation of a transverse linearization around the desired trajectories; specifically, the transverse linearization is a linear system with linearized impulse effects which locally represents the original transversal dynamics of a target trajectory. Next, a receding-horizon controller is designed to exponentially stabilize the linear impulsive system. The designed controller has been verified by a walking experiment [7] over uneven ground where the height varied by steps of $2.0 \mathrm{~cm}$. A neural network was tuned to accommodate irregular surfaces in [9]. The algorithm was tested on the robot Rabbit, whose legs are $80 \mathrm{~cm}$ long, resulting in $1.5 \mathrm{~cm}$ groundheight variations being accommodated.

A sensory reflex-based control strategy has been considered for bipedal walking [10] and for running over uneven ground [11]. In response to various types of disturbance, such as tripping and slipping [11], or step-down and step-up [10], a separately designed reflex controller is activated to attenuate the effects of the disturbances. The sensory reflex control proposed in [10] was tested to accommodate obstacles $1.0 \mathrm{~cm}$ in height, while a reflex strategy in [11] was verified only in simulation to the best knowledge of the authors. A reflexbased control strategy for traversing unknown sloped terrain was presented in [12] along with experimental verification. On the other hand, prior information of the stair profile is required in order to handle stepped terrain. 
While important progress is being made on walking over uneven ground with unknown abrupt variations, significant restrictions still remain. The experimental work in [4], [5], [6], [9], [7] and [10] accommodates only obstacles that are less than $6 \%$ of leg length, a value that is small when compared to common obstacles in everyday life, such as the height of steps in a building or the curb height of a sidewalk on a city street.

In this paper, we propose a new control policy for the planar bipedal robot MABEL [13], which weighs $65 \mathrm{~kg}$ and has $1 \mathrm{~m}$-long legs. The control policy allows MABEL to traverse various ground profiles, including ascent of a stair with a height of $12.5 \mathrm{~cm}$ (12.5\% of the leg length), step-down from a platform with a height of $18.5 \mathrm{~cm}$ (18.5\% of the leg length), and walking over constructed platforms which consist of steps with heights of $10.5 \mathrm{~cm}$ and $8.0 \mathrm{~cm}$ (see Figure 1), without falling. The robot is provided information on neither where the change in height occurs, nor by how much.

The remainder of the paper is organized as follows. Section II describes the general features of MABEL's morphology, and summarizes two mathematical models for a walking gait. Section III provides the design of the baseline controller reported in [14] and an initial step-down experiment reported in [15]. Individual control designs to accommodate various types of obstacles including step-down, step-up, and tripping are presented in Section IV. Section V introduces a finite-state machine to manage the transitions among these controllers. In Section VI, the overall controller is verified on a detailed simulation model reported in [15]. Experimental results of the new controller are provided in Section VII. Finally, Section VIII provides concluding remarks and briefly discusses future research plans.

\section{Hardware And Mathematical Model of MABEL}

This section briefly introduces MABEL, the robot used to test the proposed finite-state machine, and two mathematical models for control law design and verification. This material is based primarily on [14], [13], [15].

\section{A. Description of MABEL's hardware}

MABEL is a planar bipedal robot comprised of five rigid links assembled to form a torso and two legs with knees. As shown in Figure 2, the legs are terminated in point feet. All actuators are located in the torso, so that the legs are kept as light as possible. Unlike most bipedal robots, the actuated degrees of freedom of each leg do not correspond to the knee and hip angles. Instead, for each leg, a collection of cabledifferentials is used to connect two motors to the hip and knee joints in such a way that one motor controls the angle of the virtual leg consisting of the line connecting the hip to the toe, and the second motor is connected in series with a spring in order to control the length or shape of the virtual leg (see Figure 3). The reader is referred to [16], [13], [15] for further details on the transmission. Table I summarizes the convention used for subscripts for variables in the remainder of the paper.

The springs in MABEL serve to isolate the reflected rotor inertia of the leg-shape motors (see Figure 3) from the impact

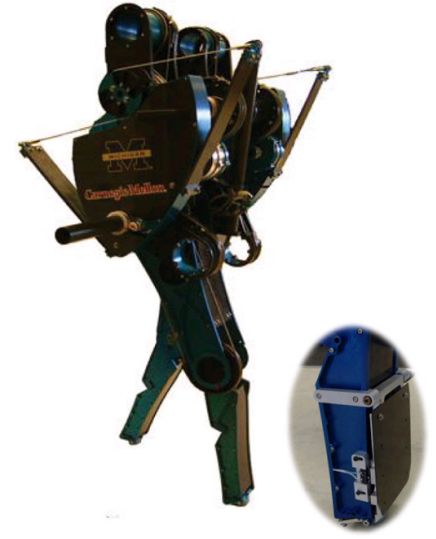

(a)

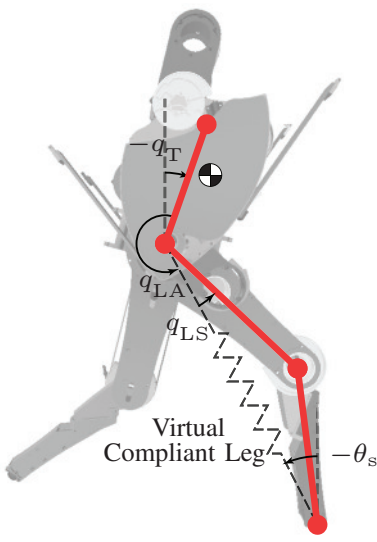

(b)
Fig. 2: (a) MABEL is shown with the switch in front of the shin that is used to detect contact with obstacles. The robot is planar, with a boom providing stabilization in the frontal plane. The robot weighs $65 \mathrm{~kg}$ and is $1 \mathrm{~m}$ at the hip. (b) The virtual compliant leg created by the drivetrain through a set of differentials.

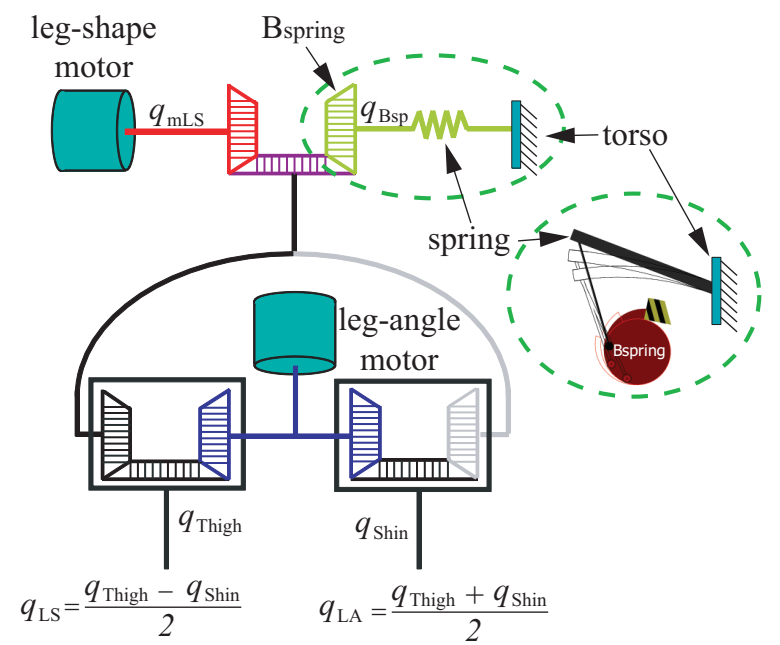

Fig. 3: MABEL's powertrain (same for each leg), all housed in the torso. Two motors and a spring are connected to the traditional hip and knee joints via three differentials, which are connected such that the actuated variables are leg angle and leg shape (see Figure 2), and so that the spring is in a series with the leg-shape motor. The differentials are realized with pulleys and cables; for further details, see [15].

forces at leg touchdown and to store energy in the compression phase of a walking gait, when the support leg must decelerate the downward motion of the robot's center of mass. The energy stored in the spring can then be used to redirect the center of mass upwards for the subsequent phase. These properties (shock isolation and energy storage) enhance the energy efficiency of walking and reduce the overall actuator power requirements [14], [17].

As depicted in Figure 2a, MABEL is equipped with contact switches installed on the bottom of the toe and the front of the shin. The contact switch installed on the bottom of the toe is used for detecting impacts with the ground whereas the contact switch installed on the front of the shin is used for detecting contact with obstacles. These contact switch signals, 
TABLE I: Nomenclature for subscripts.

\begin{tabular}{cl}
\hline Subscript & Variable \\
\hline LA & Leg Angle \\
LS & Leg Shape \\
mLA & Motor Leg Angle \\
mLS & Motor Leg Shape \\
T & Torso \\
Bsp & B $_{\text {spring Pulley Angle }}$ \\
st & Stance leg \\
sw & Swing leg \\
\hline
\end{tabular}

along with position encoder signals located on the joints, will be used in the design of a finite-state machine.

\section{B. Mathematical Model}

Two models of MABEL have been developed and identified in [14] and [15]. This section briefly summarizes a simplified design model that is appropriate for control design and a more detailed model appropriate for controller verification. These two models will be used extensively in Section IV. The simplified design model will be used for iterative controller design because it can be simulated twenty times faster than the detailed model. The detailed model will be used to verify controller performance because it better reflects the actual hardware.

1) Simplified Design model: The hybrid model consists of a continuous-time stance phase and an instantaneous double support phase. The generalized coordinates are taken as,

$$
q_{\mathrm{s}}:=\left(q_{\mathrm{LA}_{\mathrm{st}}} ; q_{\mathrm{mLS}_{\mathrm{st}}} ; q_{\mathrm{Bspst}_{\mathrm{st}}} ; q_{\mathrm{LA}_{\mathrm{sw}}} ; q_{\mathrm{mLS} \mathrm{sw}} ; q_{\mathrm{T}}\right),
$$

where, as in Figure 2 and Figure 3, $q_{\mathrm{LA}_{\mathrm{st}}}, q_{\mathrm{mLS}_{\mathrm{st}}}$, and $q_{\mathrm{Bspst}}$ are the leg angle, leg-shape motor position, and angle of the pulley $B_{\text {spring }}$ (a pulley which is connected to the free end of the spring as shown in Figure 3, and therefore corresponds to spring deflection) of the stance leg, respectively; $q_{\mathrm{LA}_{\mathrm{sw}}}$ and $q_{\mathrm{mLS}}$ are the leg angle and leg-shape motor position of the swing leg, respectively; $q_{\mathrm{T}}$ is the angle of torso with respect to the vertical. With the generalized coordinates defined in (1), the equations of motion of the continuous-time stance phase are obtained using the method of Lagrange [18].

An impact occurs when the swing leg touches the ground, and is modeled here as an inelastic contact between two rigid bodies. It is assumed that there is neither rebound nor slip at impact. The method of [19] provides a (static) map that takes the state variables $x_{\mathrm{s}}:=\left(q_{s} ; \dot{q}_{s}\right)$ just before impact to their values just after impact when impact occurs.

More details about the development of the impact map and the hybrid model for MABEL are presented in [14], [15].

2) Detailed Model: The simplified design model does not fully reflect experimental reality due to the following reasons: cable stretch in the robot's drivetrain; asymmetry due to the boom radius not being large enough; the fact that the simplified impact model assumes an instantaneous double support phase whereas, in experiments, the double support phase lasts approximately $20 \mathrm{~ms}$. More details are provided in [14] and [15]. By representing cable stretch as a springdamper, incorporating the boom dynamics to account for asymmetry side-to-side, and computing ground reaction forces on the basis of a compliant ground model and a LuGre friction model [20], [21], the accuracy of the model is significantly

improved. However, because of the complexity of this model, simulations of the detailed model take 20 times longer than the simplified design model. Hence, this model is not appropriate for optimization processes which may require thousands of simulations.

Therefore, to take advantage of each model's strengths, which are low computational effort for the simplified design model and high accuracy for the detailed model, iterative controller design is conducted on the simplified design model, and then the designed controller is tested on the detailed model before implementing it on the robot.

\section{BASEline CONTROLLER AND SHOCK-ABSORBING CONTROLler}

\section{A. Baseline Controller}

MABEL's baseline feedback controller is designed using the method of virtual constraints [22]. The particular controller used here has been reported in [14]. The method of virtual constraints begins with the choice of outputs which depend on only configuration variables and take the form

$$
y=h\left(q_{\mathrm{s}}\right)=h_{0}\left(q_{\mathrm{s}}\right)-h^{d}\left(s\left(q_{\mathrm{s}}\right), \alpha\right) .
$$

In the baseline controller, the controlled variables are

$$
h_{0}\left(q_{\mathrm{s}}\right)=\left[\begin{array}{c}
q_{\mathrm{mLS}} \\
q_{\mathrm{LA}} \\
q_{\mathrm{mLS}} \\
q_{\mathrm{T}}
\end{array}\right]
$$

and $h^{d}\left(s\left(q_{\mathrm{s}}\right), \alpha\right)$ is a vector representing the desired evolution of the controlled variables as a function of $s\left(q_{\mathrm{s}}\right)$, a scalar function of the configuration variables that replaces time in a standard tracking controller. The function $s\left(q_{\mathrm{s}}\right)$ is designed to be strictly monotonically increasing over the course of a step, and is selected as

$$
s\left(q_{\mathrm{s}}\right)= \begin{cases}\frac{\theta\left(q_{\mathrm{s}}\right)-\theta^{+}}{\theta^{-}-\theta^{+}}, & \theta^{+}<\theta\left(q_{\mathrm{s}}\right)<\theta^{-} \\ 1, & \theta\left(q_{\mathrm{s}}\right) \geq \theta^{-} \\ 0, & \theta\left(q_{\mathrm{s}}\right) \leq \theta^{+}\end{cases}
$$

where $\theta$ is the absolute angle formed by the virtual compliant leg relative to the ground, that is,

$$
\theta\left(q_{\mathrm{s}}\right)=\pi-q_{\mathrm{LA}_{\mathrm{st}}}-q_{\mathrm{T}},
$$

and $\theta^{+}$and $\theta^{-}$are the values of $\theta\left(q_{\mathrm{s}}\right)$ at the beginning and end of a step, respectively.

If a feedback can be found such that the output $y$ is driven asymptotically to zero, then the solutions of the closed-loop system asymptotically satisfy $h\left(q_{\mathrm{s}}\right)=0$, which has the form of a holonomic constraint on a mechanical system (for additional information about virtual constraints, see [23]).

In the baseline controller, the desired evolution of the controlled variables in (3) is specified by the functions $h_{\mathrm{mLS}_{\mathrm{st}}}^{d}$, $h_{\mathrm{LA}}^{d} A_{\mathrm{sw}}, h_{\mathrm{mLS}_{\mathrm{sw}}}^{d}$, and $h_{\mathrm{T}}^{d}$, respectively which are parametrized $5^{\text {th }}$-order Bézier polynomials with corresponding Bézier Coefficients $\alpha_{\mathrm{mLS}_{\mathrm{st}}}, \alpha_{\mathrm{LA}_{\mathrm{sw}}}, \alpha_{\mathrm{mLS}}$, and $\alpha_{\mathrm{T}}$. The desired evolution 
is assembled as

$$
h^{d}(s, \alpha)=\left[\begin{array}{cc}
h_{\mathrm{mLS}}^{d}(s, \alpha) \\
h_{\mathrm{LA}}^{d}(s, \alpha) \\
h_{\mathrm{mLS}_{\mathrm{sw}}}^{d}(s, \alpha) \\
h_{\mathrm{T}}^{d}(s, \alpha)
\end{array}\right],
$$

where $\alpha$ is organized as,

$$
\alpha=\left[\alpha_{\mathrm{mLS}_{\mathrm{st}}}, \alpha_{\mathrm{LA}_{\mathrm{sw}}}, \alpha_{\mathrm{mLS}_{\mathrm{sw}}}, \alpha_{\mathrm{T}}\right]^{T} .
$$

How the functions in $h^{d}(s, \alpha)$ are constructed from Bézier polynomials and how the parameters are chosen to create a periodic walking gait in the closed-loop system are both explained in [22] and [14].

In principle, the virtual constraints can be implemented on the robot by any feedback capable of driving $y$ to zero. In the experiments described below, we use the feedforward-plusPD-controller,

$$
u^{P D}(x)=u^{*}\left(s\left(q_{\mathrm{s}}\right), \alpha\right)-K_{P} y-K_{D} \dot{y},
$$

where $u^{*}\left(s\left(q_{\mathrm{s}}\right), \alpha\right)$ is the nominal torque along the periodic orbit determined from the parameter-optimization problem when designing the virtual constraints, and $y$ is defined in (2). The values of $K_{P}$ and $K_{D}$ were obtained by hand tuning, and are given by

$$
K_{P}=\operatorname{diag}\left(\begin{array}{llll}
6 & 45 & 6 & 45
\end{array}\right), K_{D}=\operatorname{diag}\left(\begin{array}{llll}
0.125 & 1 & 0.125 & 1
\end{array}\right) .
$$

The above process results in the virtual constraints which correspond to the nominal walking gait presented in [14], with an average walking speed of approximately $1.0 \mathrm{~m} / \mathrm{s}$. Here, we modify the nominal virtual constraints so that the end of the swing leg at mid-stance can clear a $3 \mathrm{~cm}$ obstacle, allowing the robot to step onto a platform before stepping off it. Henceforth, we call this the baseline controller.

\section{B. Baseline step-down performance}

As reported in [15], using the control law (8) and the baseline virtual constraints, MABEL can accommodate a 2.0 inch $(5.08 \mathrm{~cm})$ step-down disturbance. However, MABEL fell after stepping off the 2.5 inch platform because the leg broke on the ensuing step; the video is available at [24].

Further analysis of the experimental results reveals that the feedback system overreacts when correcting the forwardpitching motion of the torso arising from the impact at stepdown. Moreover, this overreaction causes a second, very rapid, forward-pitching motion of the torso. Because the angle of the swing leg was controlled relative to the torso, the swing leg rotated forward rapidly as well and impacted the ground with sufficient force to break the leg. Though not reported in [15], the experiment was repeated several times, with the same result, namely, a broken leg following a 2.5 inch stepdown.

\section{Active Force Control and Virtual Compliance for Shock Absorption}

To attenuate excessive torso pitching following a large stepdown, we adopted the idea of a switching controller from [25], along with active force control [26]. The height of the platform, or equivalently, the depth of the step down, can be immediately computed at impact from the lengths of the robot's legs and the angles of its joints. If the calculated height of the platform is greater than $3 \mathrm{~cm}$, the baseline controller is replaced for one step with a controller whose purpose is to attenuate pitching of the torso from the step-down disturbance. Then, at the beginning of the very next step, the baseline controller is re-applied.

The new controller, called active force control, imposes virtual holonomic constraints on only three variables, $q_{\mathrm{LA}_{\mathrm{sw}}}, q_{\mathrm{mLS}_{\mathrm{sw}}}$, and $q_{\mathrm{T}}$, instead of four variables, as in the baseline controller. In particular, the system input corresponding to the stance motor leg shape is left free and is not used for imposing a virtual constraint. Recall that this motor is in series with a physical spring in the drivetrain, as shown in Figure 3. Building on an idea developed in [27], [26] for bipedal running on MABEL, we use the torque input of this motor to create an additional virtual compliant element by defining the feedback,

$$
u_{\mathrm{mLS}_{\mathrm{st}}}(x)=-k\left(q_{\mathrm{mLS}}-q_{\mathrm{rest}}\right)-b\left(\dot{q}_{\mathrm{mLS}_{\mathrm{st}}}\right) .
$$

For further use, we assemble the independent parameters of the virtual compliance as $\alpha_{\mathrm{vc}}=\left[k, b, q_{\text {rest }}\right]^{T}$.

This feedback essentially turns the motor leg shape into a shock absorber with stiffness $k$, damping $b$, and rest position $q_{\text {rest }}$. As will be seen, this method of creating a virtual compliant element serves to maintain good ground contact forces (the friction cone is respected and the normal component is positive) during large step-down events. Parameters in the controller were obtained by solving a constrained optimization problem, and the proposed switching controller resulted in a dramatic increase in performance: MABEL was repeatedly able to step off an 8.0 inch $(20.32 \mathrm{~cm})$ platform without falling [28].

\section{Controller Design for Large UneXPeCted Ground HeIGHT InCREASES AND DECREASES}

\section{A. Motivation of the Control Design}

As established in the successful $20 \mathrm{~cm}$ step-down experiments in Section III, by activating the specifically designed step-down controller in the form of active force control, in response to a step-down disturbance, MABEL could not only maintain a positive vertical ground reaction force, causing the stance toe to stay in contact with the ground, but also reduce torso oscillation by a significant amount, thereby attenuating the impact force on the swing toe at the end of the ensuing step of the step-down event.

It appears that, similar to MABEL's switching control strategy for a step-down disturbance, humans also change their gait strategy in response to disturbances in ground height, in upslope as well as down-slope walking [29], [30], [31]. Research studies have shown that the typical level walking gait may require small modifications for small grade ramps; however, steeper grades seem to require significant changes in the gait patterns to accommodate both large up-slope changes and large down-slope changes. Along with modifications of gait patterns, it is found that humans adjust the stiffness of the stance leg in response to ground variations in height during running [32], 
[33]. The adjustment is characterized by an increase in leg stiffness in preparation for a step-down disturbance, and a reduction in response to a step-up disturbance.

Tripping over obstacles is also a commonly occurring perturbation while a human is walking, and it is shown in [34], [35] that humans accommodate a tripping disturbance by changing their gait strategy. Cutaneous receptors are stimulated when the trajectory of the swing foot is obstructed, and a response which ensures the removal of the limb from the perturbation, as well as the safe continuation of the locomotor pattern, is activated to deal with the detected perturbation.

In MABEL, this idea of a reflex strategy in response to ground-height variations and tripping perturbations can be implemented in the context of switching-control design and active force control. Step-up and step-down disturbances can be detected based on calculated swing heights and the contact switch signal on the toe at the moment of impact. Furthermore, MABEL can also detect tripping over obstacles by using the signals of the switch located in front of the shin and at the end of toe, replacing cutaneous receptors in humans.

For each type of disturbance, gait pattern and leg stiffness can be adjusted by switching to a specifically designed controller. Changes in the effective leg stiffness can be made by varying the values of stiffness $k$, damping $b$, and rest position $q_{\text {rest }}$, and adjustments in gait pattern can be made by redesigning the virtual constraints. In the design of the controller, stiffness, damping, and rest position are the design parameters to be determined along with virtual constraints for the rest of the controlled variables. Furthermore, drawing on the observation in [36] that a human's stance knee essentially shows the dynamic behavior of a spring-damper system when walking on flat ground, we also extend the use of active force control to walking over flat ground.

Transitions among the designed controllers for flat-ground walking, stepping-down, stepping-up, and a trip-reflex will be managed by a finite-state machine, which is presented in Section V.

\section{B. Summaries of Controller Designs}

The controllers for flat-ground walking, stepping-down, stepping-up, and a trip-reflex are called Regular-walking (RW), Step-down (SD), Step-up (SU), and Tripping-reflex (TR) controllers, respectively. This section summarizes the design process of each controller.

1) Regular-Walking Controller: The regular-walking controller is obtained from the baseline controller by replacing its motor leg shape control with a virtual compliance to include active force control. The parameter values for the virtual compliance $\alpha_{\mathrm{vc}}$ are iteratively found through simulations, and given by,

$$
\alpha_{v c}=\left[\begin{array}{lll}
1.8, & 0.1297, & 2.7
\end{array}\right]^{T} .
$$

The regular-walking controller retains the virtual constraints of the baseline controller for the torso, swing leg angle, and swing leg shape.

2) Step-down and Step-up Controllers: As explained in Section III, excessive oscillation in the torso angle after a

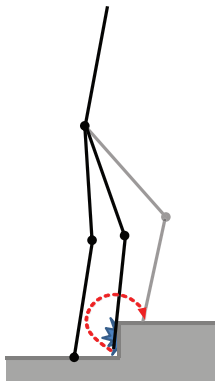

(a)

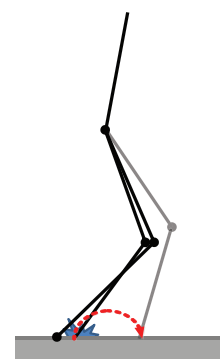

(b)

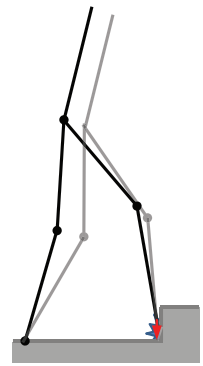

(c)
Fig. 4: Various situations of tripping. The red line with an arrow at the end illustrates an example swing foot trajectory in response to the tripping event. (a) Early tripping over obstacles. (b) Premature (i.e., early) contact with the ground. (c) Late tripping over obstacles.

step-down event was the main reason for the robot's falling. Therefore, in the design of the step-down controller, it is important to redesign the virtual constraint for the torso to reduce the oscillation. When redesigning the virtual constraint for the torso, the first and last coefficients of the Bézier polynomials from the baseline controller are retained in order to provide a smooth transition from and to the regular-walking controller. The coefficients between them, denoted hereafter by $\alpha_{\mathrm{T}}^{2 \cdots 5} \in \mathbb{R}^{4}$, together with the parameter values for the virtual compliance $\alpha_{\mathrm{vc}}$ will be selected through optimization as explained in Section IV-C.

In the case of step-up disturbances, simulation of the baseline controller shows that oscillations in the vertical ground reaction force is the main reason leading to falls instead of the excessive torso oscillation. Therefore, the baseline virtual constraint for the torso is retained whereas parameter values for the virtual compliance $\alpha_{\mathrm{vc}}$ are redesigned through optimization.

3) Tripping-reflex controller: Tripping occurs when the robot's swing foot experiences unexpected impacts. The impacts can arise due to obstacles, as shown in Figures 4a and 4c, or due to inadequate foot height, as shown in Figure 4b. The required response varies with the nature of the impact and it is thus important that the robot has adequate sensing to differentiate among the cases shown in Figure 4. For this purpose, MABEL has contact switches on the front of each lower shin, as shown in Figure 2a, in addition to the traditional contact switches on the end of each leg.

It has been suggested that humans adopt two different strategies for recovery from tripping, depending on whether it occurred in the early or late swing phase [34], [35]. A strategy of rapidly elevating the swing limb is activated in response to early tripping while a lowering strategy is used in response to late tripping. Similar strategies are applied here.

Reflexive Strategy for Late Tripping: Condition for late tripping $\left(\mathcal{S}_{\mathrm{RL}}\right)$ can be defined mathematically with respect to the robot's state and contact switch signals. If the frontal surface of the swing foot hits an obstacle $\left(s w_{\mathrm{sh}}=1\right)$ with the step being close to the end $\left(s\left(q_{\mathrm{s}}\right) \geq s_{\text {early }}\right)$ as well as the absolute swing leg angle being advanced sufficiently $\left(q_{\mathrm{LA}}^{a b s}:=q_{\mathrm{LA}} A_{\mathrm{sw}}+q_{\mathrm{T}} \geq q_{\text {early }}\right)$ to yield the next step starting with a value of $s\left(q_{\mathrm{s}}\right)$ close to 0 , then tripping is classified as 


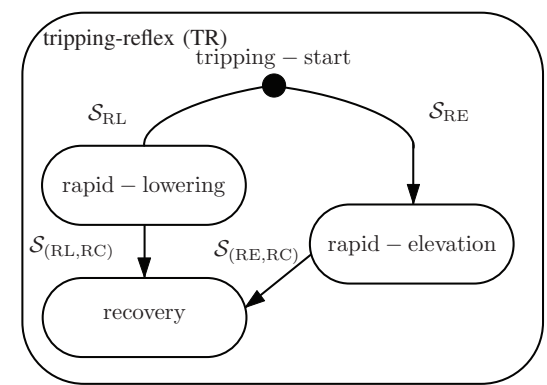

Fig. 5: Finite-state machine of tripping-reflex controller

late tripping. In this paper, $s_{\text {early }}$ and $q_{\text {early }}$ are set to 0.66 and $190 \mathrm{deg}$.

In response to late tripping, the rapid-lowering $(\mathrm{RL})$ strategy of the swing leg is applied. The controller is not changed until the swing foot touches the ground. Because all of the walking gaits used in this paper are designed to lower the swing foot near the end of the gait to initiate leg swapping, the swing leg will be lowered making contact with the ground, and, at the ensuing step, a recovery controller focusing on rejecting the tripping disturbance of the previous step is applied. The parameters of the virtual compliance $\alpha_{\mathrm{vc}}$, and coefficients of the Bézier polynomials for the torso virtual constraints except for the last coefficient, $\alpha_{\mathrm{T}}^{1 \cdots 5} \in \mathbb{R}^{5}$, are chosen by optimization as explained in Section IV-C. The last coefficient of the torso virtual constraint for the baseline controller is retained in order to smooth the transition to the regular-walking controller.

Reflexive Strategy for Early Tripping: Condition for early tripping $\left(\mathcal{S}_{\mathrm{RE}}\right)$ can be defined mathematically with respect to the robot's state and contact switch signals. Referring to Figure 4 , impact with the ground $\left(s w_{\text {toe }}=1\right)$ or an obstacle $\left(s w_{\mathrm{sh}}=1\right)$ is defined to be early tripping if it occurs before or during the middle of the gait $\left(s\left(q_{\mathrm{s}}\right)<s_{\text {early }}\right)$ or with swing leg not advanced adequately (absolute swing leg angle being less than some value, that is, $\left.q_{\mathrm{LA}}^{a b s}:=q_{\mathrm{LA}_{\mathrm{sw}}}+q_{\mathrm{T}}<q_{\mathrm{early}}\right)$.

In response to early tripping, the rapid-elevation (RE) strategy of the swing leg is activated. Rapid-elevation of the swing leg is accomplished by rapidly bending the swing knee, which is in turn accomplished by repositioning the leg shape motor of the swing leg. The motor's position is set through the appropriate choice of $h_{\mathrm{mLS}_{\mathrm{sw}}}^{d}\left(\theta\left(q_{\mathrm{s}}\right), \alpha\right)$ in (6), designed so that the swing leg will clear a $10 \mathrm{~cm}$ obstacle. Examples of modification are given in Figure 6. The purpose of the elevation strategy of the swing leg is to place the swing foot on top of the obstacle, and allow the robot to continue the walking gait. Once the swing foot is on top of the obstacle, the robot can continue walking by applying the step-up controller at the ensuing step.

If the swing foot gets trapped against the vertical step and fails to be placed on top of the obstacle at the end of the step in spite of the rapid-elevation of the swing leg, the recovery controller, which is designed in Section IV-B3, will be applied at the ensuing step in order to have a safe continuation of the walking gait. Failure of the rapid-elevation strategy of the swing leg can be detected by checking the calculated height of the swing foot at the end of the gait, denoted by $p_{\text {toe }}^{v}\left(q_{\mathrm{s}}\right)$, and by how much the swing foot advanced horizontally before it touches the ground, denoted by $\delta p_{\text {toe }}^{h}$. If the swing foot
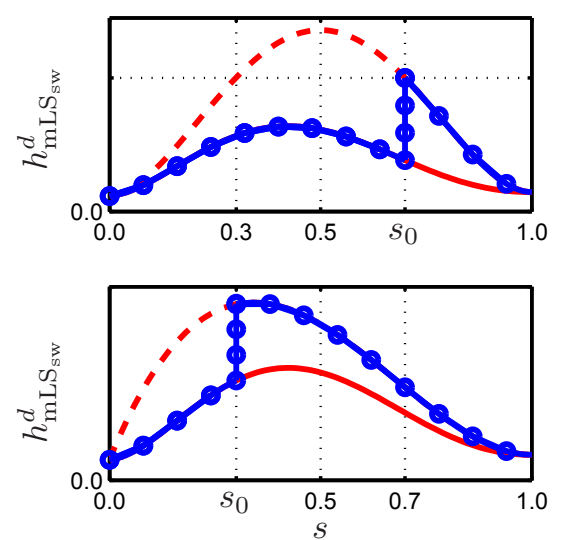

Fig. 6: Example of modification on the desired trajectory of the motor leg shape. Blue lines with circle indicate modified desired trajectory for the motor leg shape. Solid red line indicates original desired trajectory of the motor leg shape while dashed red line shows elevated desired trajectory. $s_{0}$ is the value of $s$ at the moment of tripping. Graph on top: when $s\left(q_{\mathrm{s}}\right)>s_{0}$, Graph on bottom: when $s\left(q_{\mathrm{s}}\right) \leq s_{0}$

gets stuck on the vertical step, the swing foot will not be able to advance forward $\left(\delta p_{\text {toe }}^{h} \approx 0\right)$ and will make an impact with the ground instead of on top of the obstacle $\left(\left|p_{\text {toe }_{\text {sw }}}^{v}\left(q_{\mathrm{s}}\right)\right| \leq \Delta H\right)$. Here, $\Delta H \in \mathbb{R}$ is a scalar threshold value, which is set to $0.05 \mathrm{~m}$ in this paper. Combining all of these conditions yields switching condition for rapid-elevation controller to recovery controller $\left(\mathcal{S}_{(\mathrm{RE}, \mathrm{RC})}\right)$.

The overall tripping-reflex controller is summarized in Figure 5 including rapid-lowering and rapid-elevation of the swing leg in response to late and early tripping, and activation of the recovery controller after the failure of rapid-elevation of the swing leg strategy.

\section{Determining Controller Parameters}

1) Process overview:: The parameters for the step-down, step-up, and recovery controllers are obtained through a two stage process. In the first stage, the controller parameters for the torso and stance leg are obtained by solving a constrained optimization problem. Denote the collection of these parameter values by $\Theta^{\mathrm{SD}, \mathrm{SU}, \mathrm{RC}}$. In the second stage, the virtual constraints for the swing leg shape $\alpha_{\mathrm{mLS}_{\mathrm{sw}}}$ and angle $\alpha_{\mathrm{LA}}$ are re-designed by hand, for each of the controllers, to ensure foot clearance (e.g., prevent premature toe stubbing).

Because the legs are relatively light, swing leg movement only weakly affects the robot's overall dynamics; consequently, hand tuning of the parameters of the swing leg does not significantly change the optimization results of the first stage of parameter selection. To confirm this, both the optimized parameters and the hand-tuned parameters are verified via simulation to satisfy the constraints used in the the constrained optimization problem with the optimization cost not being changed appreciably.

Table II summarizes the design parameters for the regularwalking, step-down, step-up, and tripping-reflex controllers. The detailed optimization process to obtain the design parameters is provided next.

2) Process details:: The optimization problems for the selection of $\Theta^{\mathrm{SD}, \mathrm{SU}, \mathrm{RC}}$ are posed as follows. Assume the robot is 


\begin{tabular}{lcc}
\hline & Optimization & Hand-tuning \\
\hline Regular-walking Controller & $\cdot$ & $\alpha_{\mathrm{vc}}$ \\
Step-Down Controller & $\alpha_{\mathrm{vc}}, \alpha_{\mathrm{T}}^{2 \cdots 5}$ & $\cdot$ \\
Step-Up Controller & $\alpha_{\mathrm{vc}}$ & $\alpha_{\mathrm{LA}_{\mathrm{sw}}}, \alpha_{\mathrm{mLS}}$ \\
Recovery Controller & $\alpha_{\mathrm{vc}}, \alpha_{\mathrm{T}}^{1 \cdots 5}$ & $\alpha_{\mathrm{LA}}$ \\
\hline
\end{tabular}

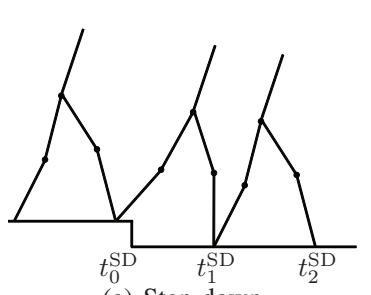

(a) Step-down

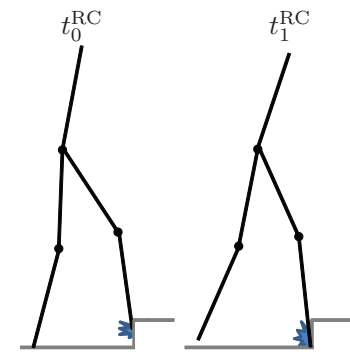

(c) Recovery

Fig. 7: Timeline for controller design.

on the periodic orbit corresponding to the baseline controller, and hence is walking on flat ground. As displayed in Figure 7 , let $t_{0}^{\mathrm{SD}, \mathrm{SU}, \mathrm{RC}}$ be the time that the robot is at the end of the last step on flat ground, let $t_{1}^{\mathrm{SD}, \mathrm{SU}, \mathrm{RC}}$ be the moment that the stepdown, step-up, or recovery controller is activated in response to step-down, step-up, or late tripping, and let $t_{2}^{\mathrm{SD}, \mathrm{SU}, \mathrm{RC}}$ denote the end of the step with the step-down, step-up, or recovery controller. Over the interval $\left[t_{1}^{\mathrm{SD}, \mathrm{SU}, \mathrm{RC}}, t_{2}^{\mathrm{SD}, \mathrm{SU}, \mathrm{RC}}\right]$, the robot is operating under the controller to be designed. For step-down and step-up controllers, once the step-down depth or step-up height is specified, $x\left(t_{1}^{\mathrm{SD}, \mathrm{SU}}\right)$, the state of the robot at time $t_{1}^{\mathrm{SD}, \mathrm{SU}}$, is known. In the case of late tripping, because the swing foot slips down along the wall of the obstacle over the interval $\left[t_{0}^{\mathrm{RC}}, t_{1}^{\mathrm{RC}}\right)$ while the stance foot is on the ground, the trajectory cannot be simulated using the simplified design model, because it does not account for double-support dynamics. Therefore, the detailed model is used for the simulation of this slip behavior during double support, and the value of the robot's state at $t_{1}^{\mathrm{RC}}$ after the impact with the ground, $x\left(t_{1}^{\mathrm{RC}}\right)$, is acquired from the detailed model simulation data. As shown in Figure $7 \mathrm{c}$, the time at the end of the step with the recovery controller varies depending on the sizes of the obstacles. In this optimization problem, the following two scenarios are considered. In the scenario a, the robot has tripped over a $10 \mathrm{~cm}$ high obstacle and the swing foot is supported on the top of the obstacle at the end of the step. We define $t_{2 a}^{\mathrm{RC}}$ to be the time when the swing foot touches the top of the obstacle. In the scenario b, we assume that the robot has tripped over a narrow obstacle, thus the swing foot crosses over the obstacle and touches the ground at the end of the step. We define $t_{2}^{\mathrm{RC}}$ to be the time when the swing foot touches the ground as shown in Figure 7c.
Numerical optimization problems for step-down, step-up, and late tripping are now posed so that the trajectory under the controller can be continued in such a way that the robot will not fall. In principle, the optimization could consider several steps, but only one step is considered here.

Objective: Select $\Theta^{\mathrm{SD}, \mathrm{SU}, \mathrm{RC}}$ to minimize peak-to-peak amplitude of torso oscillation as defined by,

$$
J_{\Theta^{i}}=\max _{t \in\left[t_{1}^{i}, t_{2}^{i}\right]}\left\{q_{\mathrm{T}}(t)\right\}-\min _{t \in\left[t_{1}^{i}, t_{2}^{i}\right]}\left\{q_{\mathrm{T}}(t)\right\}, i \in\{\mathrm{SD}, \mathrm{SU}, \mathrm{RC}\}
$$

where $q_{\mathrm{T}}(t)$ is trajectory of the torso angle. The cost function is optimized subject to the following constraints.

\section{Constraints for Step-down Controller, $\mathrm{CON}^{\mathrm{SD}}$ :}

1) positive horizontal swing toe position at the end of the step,

$$
p_{2}^{h}\left(t_{2}^{\mathrm{SD}}\right)>0
$$

2) bound on the ratio of tangential to normal ground reaction forces experienced by the stance leg end,

$$
\max _{t \in\left[t_{1}^{\mathrm{SD}}, t_{2}^{\mathrm{SD}}\right]}\left\{\frac{F_{1}^{T}(t)}{F_{1}^{N}(t)}\right\}<\mu_{s} ;
$$

3) minimum normal ground reaction force experienced by the stance leg end,

$$
\min _{t \in\left[t_{1}^{\mathrm{SD}}, t_{2}^{\mathrm{SD}}\right]}\left\{F_{1}^{N}(t)\right\}>C, \text { for some } C>0 ;
$$

4) avoid premature impact,

$$
s\left(q\left(t_{2}^{\mathrm{SD}}\right)\right)>1-\delta, \text { where } \delta \ll 1
$$

5) upper bound on the magnitude of torso angular velocity

$$
\max _{t}\left\{\left|\dot{q}_{\mathrm{T}}(t)\right|\right\}<\gamma_{\dot{q}_{\mathrm{T}}} \text {, for some } \gamma_{\dot{q}_{\mathrm{T}}}>0 .
$$

\section{Constraints for Step-up Controller, $\mathrm{CON}^{\mathrm{SU}}$ :}

1) $\mathrm{CON}^{\mathrm{SD}}$ with $t_{2}^{\mathrm{SD}}$ substituted by $t_{2}^{\mathrm{SU}}$.

2) to enforce that the robot ends the step-up with the stance leg shape nearly straight, which avoids starting the next step with a crouched configuration,

$$
q_{\mathrm{LS}}\left(t_{2}^{\mathrm{SU}}\right)<\gamma_{\mathrm{LS}} \text {, for some } \gamma_{\mathrm{LS}_{\mathrm{st}}}>0 \text {. }
$$

\section{Constraints for Recovery Controller, $\mathrm{CON}^{\mathrm{RC}}$ :}

1) $\mathrm{CON}^{\mathrm{SD}}$ with $t_{2}^{\mathrm{SD}}$ substituted by $t_{2}^{\mathrm{RC}}$.

2) magnitude of torso angular velocity at the end of the step,

$$
\left|\dot{q}_{\mathrm{T}}\left(t_{2}^{\mathrm{RC}}\right)\right|<\gamma_{\dot{q}_{\mathrm{T}, \mathrm{RC}}},
$$

in order to attenuate torso movement in preparation for impact,

3) torso angle at the end of the step bounded below by some constant $\gamma_{q_{\mathrm{T}, \mathrm{RC}}}$,

$$
q_{\mathrm{T}}\left(t_{2}^{\mathrm{RC}}\right)>\gamma_{q_{\mathrm{T}, r c}},
$$

to keep the torso from pitching forward too much at the end of the step, 

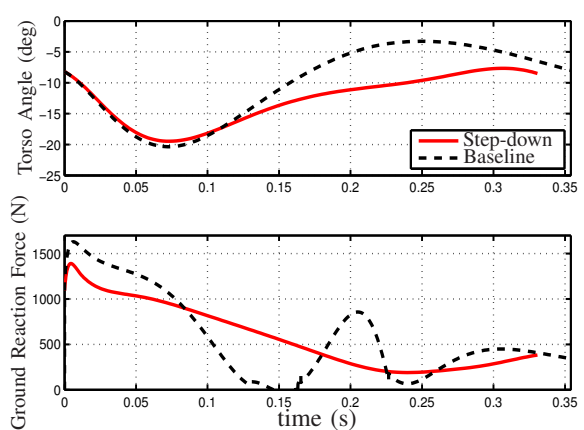

(a)
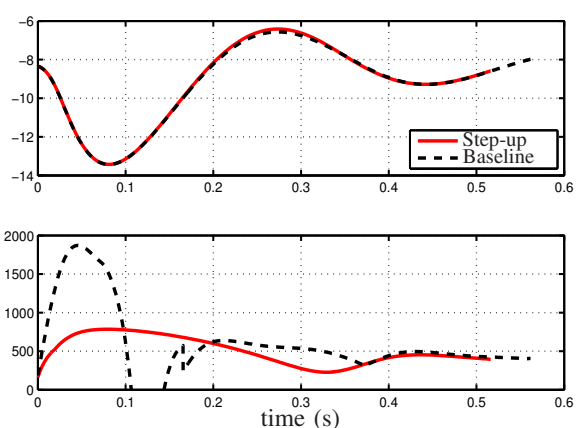

(b)
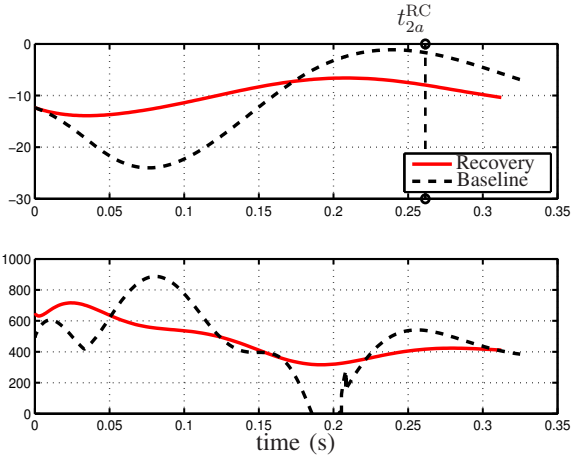

(c)

Fig. 8: Simulation data with optimal parameter vector $\Theta^{\mathrm{SD}}, \Theta^{\mathrm{SU}}$, and $\Theta^{\mathrm{RC}}$ from the simplified design model for (a) a stepdown height of $20 \mathrm{~cm}$, (b) a step-up height of $10 \mathrm{~cm}$, and (c) a trip recovery. The step-down, step-up, and recovery controllers are shown with a solid line, while dashed lines represent the baseline controller.

4) torso angular velocity at $t_{2 a}^{\mathrm{RC}}$,

$$
\dot{q}_{\mathrm{T}}\left(t_{2 a}^{\mathrm{RC}}\right) \leq 0,
$$

so that the torso has enough forward pitching velocity just before impact in order not to fall backward after an impact with a $10 \mathrm{~cm}$ high obstacle.

The optimizations are conducted over a transient phase of the gait, and thus constraints are not required to impose periodicity of an orbit. We have observed that solutions to the above optimization problems tend to steer the robot so that the configuration variables at time $t_{2}^{\mathrm{SD}, \mathrm{SU}, \mathrm{RC}}$ are near their values on the periodic orbit. We conjecture that this is because the two virtual constraints for the swing leg, as well as the ending value for the torso virtual constraint, are inherited from the baseline periodic orbit.

MATLAB's constrained optimization routine fmincon is used to perform the numerical searches outlined above ${ }^{1}$, and the resulting controllers are then applied to the simplified design model with a $20 \mathrm{~cm}$ step-down, a $10 \mathrm{~cm}$ step-up, and late tripping over a $10 \mathrm{~cm}$ platform with a length less than $80 \mathrm{~cm}$. The torso trajectory and vertical ground reaction force are shown in the top, middle, and bottom graphs of Figure 8. In Figure 8a, it is observed that the torso oscillates approximately $11 \mathrm{deg}$ during the step following the step-down. On the other hand, under the baseline controller (red dashed line), the torso noticeably overshoots while returning to the nominal lean angle. Figure $8 \mathrm{~b}$ shows in the case of step-up that there is no notable difference in the torso angle between the baseline and step-up controllers because the virtual constraints for the torso were identical. However, the vertical ground reaction force remains positive under the step-up controller while the baseline controller fails to keep this value positive, which leads to slipping. Figure $8 \mathrm{c}$ compares simulation results of the simplified design model from $t_{1}^{\mathrm{RC}}$ to $t_{2}^{\mathrm{RC}}$ for the recovery controller with optimized $\Theta^{\mathrm{RC}}$ and the baseline controller. By using the recovery controller, torso oscillation is not only reduced by approximately a factor of 3 , but also the vertical ground reaction force remains positive during the recovery

${ }^{1}$ Execution of numerical search requires the values of the constants on the right side of inequalities from (13) to (21). The specific values for MABEL used in the optimization are given in [37]. These values can be changed for other robots.
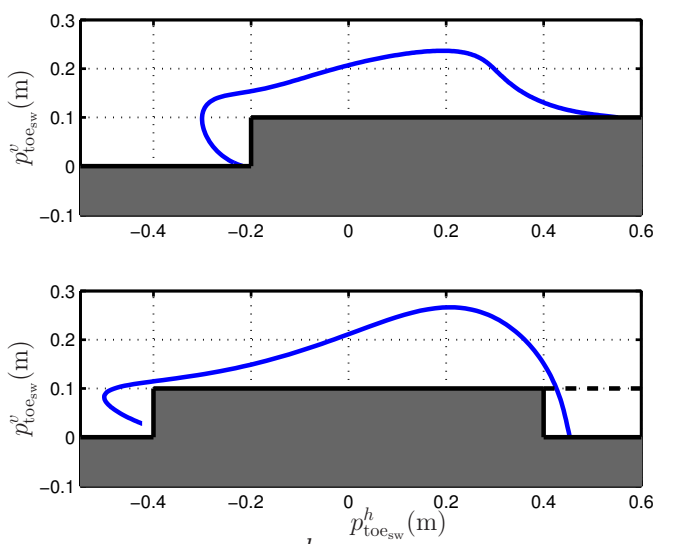

Fig. 9: Horizontal position $p_{\text {toesw }}^{h}$ and vertical position $p_{\text {toe }}^{v}$ of swing foot throughout the step. Grey colored region illustrates the vertical obstacle.

phase. Time $t_{2 a}^{\mathrm{RC}}$ is indicated by the vertical black dotted line. It is observed that at time $t_{2 a}^{\mathrm{RC}}$, the torso is pitching forward, satisfying constraint (21).

After the virtual constraints for the torso and the parameters in the virtual compliance have been chosen through optimization, the virtual constraints for the swing leg are tuned by hand for the step-up and recovery controllers. In particular, the virtual constraints for the swing leg are designed so that knee is bent rapidly by repositioning the swing motor leg shape, and the swing leg is also moved backward at the beginning of the gait by repositioning the swing leg angle motor. These actions are required to avoid re-striking the obstacle during step-up and recovery from late-tripping. Figure 9 shows the position of the swing foot throughout the step. The top graph shows the simulation result for step-up while the bottom graph shows the simulation result for late tripping. The gray colored region represents the vertical obstacle. It is observed that the swing foot neither touches the vertical step during step-up nor when recovering from late tripping.

Using both the optimized virtual compliance parameters and the hand-tuned virtual constraints for the swing leg shape and angle, we re-ran the simulations and found that all of the constraints of the constrained optimization problems were still satisfied, and the optimization cost had not changed significantly. 
TABLE III: Normalized stiffness values in response to disturbances for robot walking and human running with respect to the values used for level ground walking.

\begin{tabular}{ccc}
\hline Disturbance & Robot Walking & Human Running \\
\hline Step-Down & 1.04 & 1.09 \\
Regular-Walking & 1.00 & 1.00 \\
Step-Up & 0.89 & 0.84 \\
\hline
\end{tabular}

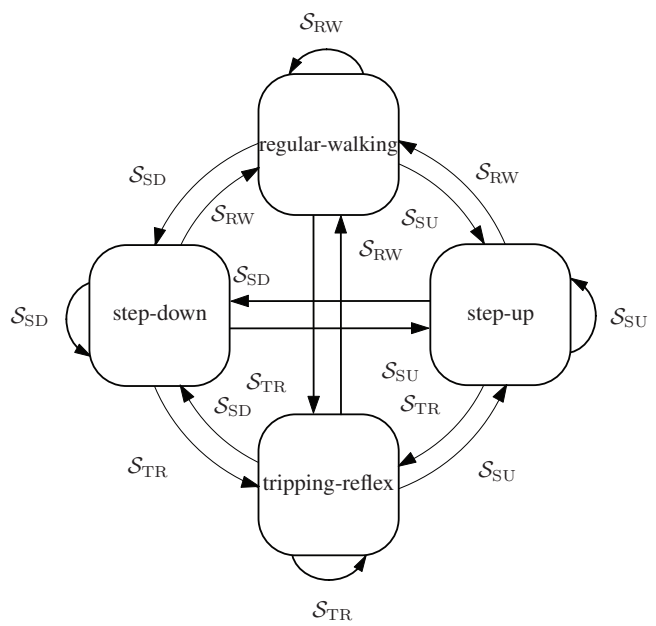

Fig. 10: Finite-state machine for managing transitions among the various contollers.

\section{Comments on the Values of Stiffness of the Virtual Com- pliant Element}

Through the optimization process explained above, stiffness values for the step-down controller, $k^{\mathrm{SD}}=2.1088$, and for the step-up controller, $k^{\mathrm{SU}}=1.2198$, were obtained. In comparison to the value for the regular-walking controller, $k^{\mathrm{RW}}=1.8$, a stiffer virtual compliance has been selected for the stepdown controller while a softer virtual compliant element has been chosen for the step-up controller. These choices of virtual compliant elements - stiffer compliance for the step-down disturbance and softer compliance for the step-up disturbance - are consistent with the strategy of humans when adjusting leg stiffness for running on uneven ground [32]. These tendencies ${ }^{2}$ are summarized in Table III.

\section{A Finite-State Machine}

This section presents a finite-state machine to manage transitions among controllers for flat-ground walking, steppingdown, stepping-up, and a trip-reflex.

The design process of the finite-state machine begins by distinguishing four types of stance phase with respect to perturbations in ground height, or disturbances from tripping over obstacles: regular-walking phase (RW), step-down phase (SD), step-up phase (SU), and tripping phase (TR), as illustrated in Figure 10. Hereafter, we index the phases by elements of the following set,

$$
W:=\{\mathrm{RW}, \mathrm{SD}, \mathrm{SU}, \mathrm{TR}\} .
$$

A decision to transition from one phase to another will be made on the basis of the values of the contact switches at the

${ }^{2}$ It should be noted that normalization of the leg stiffness values for robot walking is conducted on the effective stiffness values obtained by summing all the sources of compliance - virtual compliant elements, springs, and cable stretch - in a series manner. See Appendix A for the calculation of effective stiffness.

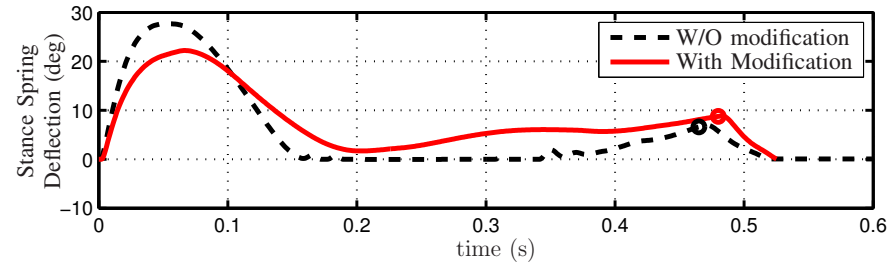

Fig. 11: Simulation data of a step with the stance knee being excessively bent at the start. The dashed line shows simulation result without modification on the virtual compliance, and the solid line illustrates simulation result with modification. The small circles on the plot indicate the end of the step.

front and end of each leg, as well as a detected change in walking surface height, which can be immediately computed at impact from the length of the robot's legs and the angles of its joints. The conditions for executing the various transitions are developed next. Firstly, the transition to RW takes place when following condition $\mathcal{S}_{\mathrm{RW}}$ is satisfied: the impact with the ground ( $x_{\mathrm{s}} \in \mathcal{S}^{H}$ ) occurs close to the end of the gait $\left(s\left(q_{\mathrm{s}}\right) \geq s_{\text {early }}\right)$ as well as when the magnitude of the height of the swing toe at the moment of the impact is less than $\Delta H$.

The transition to $\mathrm{SD}$ or $\mathrm{SU}$ occurs when the impact with the ground occurs close to the end of the gait $\left(s\left(q_{\mathrm{s}}\right) \geq s_{\text {early }}\right)$, along with the height of the swing toe at the moment of impact being less than $-\Delta H$, or larger than $\Delta H$, respectively. Those two conditions are denoted by $\mathcal{S}_{\mathrm{SD}}$ and $\mathcal{S}_{\mathrm{SU}}$.

Lastly, the transition to TR arises when the swing leg trips over obstacles or touches the ground prematurely. Tripping can be detected by checking switching condition $\mathcal{S}_{\mathrm{RL}}$ or $\mathcal{S}_{\mathrm{RE}}$.

\section{Controller Evaluation on the Detailed MODEL}

Before experimental deployment, the finite-state machine will be simulated on the detailed model. Certain straightforward modifications to the regular-walking, step-down, step-up, and tripping-reflex controllers are required due to discrepancies between the simplified and detailed models. As mentioned in Section II, the discrepancies between the simplified and detailed models mostly arise from features that are unique to MABEL, specially the robot's cable-differential-based drive train and the boom mechanism used to planarize the robot's motion. The modifications made here are therefore specific to MABEL. Initial simulations will reveal one additional modification that needs to be performed. After these changes to the controllers, the performance of the finite-state machine will be evaluated when the controllers are sequentially composed in response to various disturbances in ground height.

\section{A. Minor Modification of Controllers for Detailed Model Implementation}

As part of implementing the proposed controllers on the detailed model, the following modifications are made to compensate for the gap between the simplified design model and the detailed model.

1) Modification for Cable Stretch: The most critical reason for model discrepancy is cable stretch. To account for the 


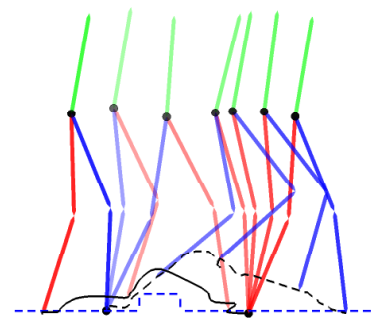

(a)
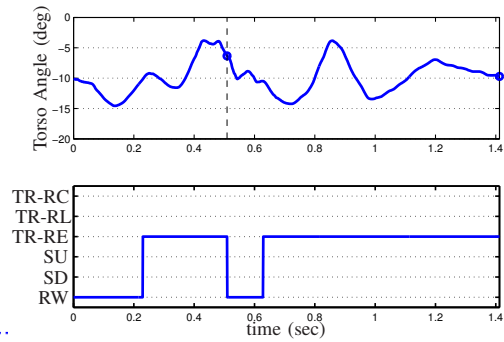

(b)
Fig. 12: Experimental data of walking over a $7 \mathrm{~cm}$ bump. (a) Stick figure illustration of the experimental data with a black circle indicating the stance toe. Black solid and dashed line represent the path of the swing leg. Swing leg modification is activated twice (see Figure 12b) to clear the leg from the obstacle. (b) Torso angle (top), $s\left(q_{\mathrm{s}}\right)$ (middle), and controller type (bottom) are shown, respectively. Blue hollow circle and vertical gray dashed line mark the end of the step.

stretching of the cables, the coefficients of the virtual compliance $k$ in regular-walking, step-down, step-up, and trippingreflex controller are modified as in [27], [26] so that the series connection of the compliance due to the cable stretch and the virtual compliance has the effective compliance specified by the optimization process. The details are given in Appendix A.

2) Modification for Asymmetry: In the experimental setup, due to the boom, the robot's hip position is constrained to lie on the surface of a sphere, rather than a plane. The hip width (distance between the legs) being 10\% of the length of the boom causes the robot to weigh $10 \%$ (almost $70 \mathrm{~N}$ ) more when supported on the inner leg than when supported on the outer leg. This causes inner-outer asymmetry in the walking gait [14]. To account for this asymmetry, the virtual compliance is made an additional $10 \%$ stiffer on the inside leg.

3) Modification for Avoiding Foot Scuffing: As discussed in Section IV, the step-up and recovery controllers use the virtual constraints for the swing leg with a modification allowing the swing leg height to be increased in order to keep the swing foot from scuffing the ground. Similar modifications are required for step-down and regular-walking controllers. Step-down Controller: When MABEL steps off platforms, the higher impact force causes an additional bend in the stance knee during the ensuing step of the step-down event. The higher the platform which MABEL steps off is, the greater the bend in the stance knee caused. To deal with this additional bend, the virtual constraint for the swing motor leg shape is increased according to the platform height calculated at impact.

Regular-walking Controller: In the regular-walking phase, foot scuffing can also occur when a step starts with the stance knee being overly bent. Therefore, an event-based control is introduced so that the virtual constraint for the swing motor leg shape is increased according to how much more the stance knee is bent from some reference value. In particular, if the stance leg shape angle is larger than $10 \mathrm{deg}$ at the start of the gait, the desired swing motor leg shape is then modified by increasing the middle two Bézier coefficients of the swing leg shape proportional to the difference between the stance leg shape angle and $10 \mathrm{deg}$.

\section{B. Additional Modification of the Virtual Compliance}

The initial simulation of the controller showed that when the stance knee angle, which is two times the leg shape angle, is bent more than $60 \mathrm{deg}$ at impact, the spring compresses further and subsequently decompresses very rapidly, causing the stance leg to lose contact with the ground. In order to account for this problem, we soften the virtual compliance by the amount by which the stance knee is additionally bent from the nominal value. The solid line in Figure 11 illustrates spring deflection after the modification. With the modification, the spring compresses and decompresses gently, and does not reach zero.

\section{Simulation of the finite-state machine}

With these modifications to the proposed controller, the simulation results show MABEL successfully traversing various ground profiles representing large obstacles without a priori information; in particular, vision feedback is not used. It is observed that the robot's configuration converges to the nominal configuration within a few steps after each disturbance event, and, in response to various disturbance events, appropriate transitions among controllers occur. For detailed analysis of the verification results, see [37].

\section{EXPERIMENT}

The proposed finite-state machine is now evaluated on the robot. In the experiments, various stair-stepped platforms are placed in the robot's path to verify various executions of the finite-state machine. Because the baseline controller already allowed MABEL to cross any combination of up and down for small obstacles with a height of less than $2.54 \mathrm{~cm}$, only obstacles with a height greater than $5 \mathrm{~cm}$ are tested here. Videos are available at [38].

\section{A. Small Bump with Height of $7 \mathrm{~cm}$}

As a first test of the tripping-reflex controller, a small bump higher than the maximum vertical position of the swing foot was placed in the middle of MABEL's walking path. As shown in Figure 12, in response to tripping over this small bump, the tripping-reflex controller was activated, and modification of the swing foot trajectory was made successfully, thereby providing clearance of the bump.

\section{B. Step-up and Step-down of $10.5 \mathrm{~cm}$ platform}

In this experiment, MABEL stepped upon a platform with a height of $10.5 \mathrm{~cm}$ (more than $10 \%$ of its leg length), walked three steps on level ground on top of the platform, and stepped off the platform; see Figure 13. Two laps were completed before the robot was stopped by a researcher. On the first lap, MABEL's swing foot struck obstacles at a late point of the step, so the rapid-lowering controller was activated in response to the late perturbation, as shown in Figure 14(a). 

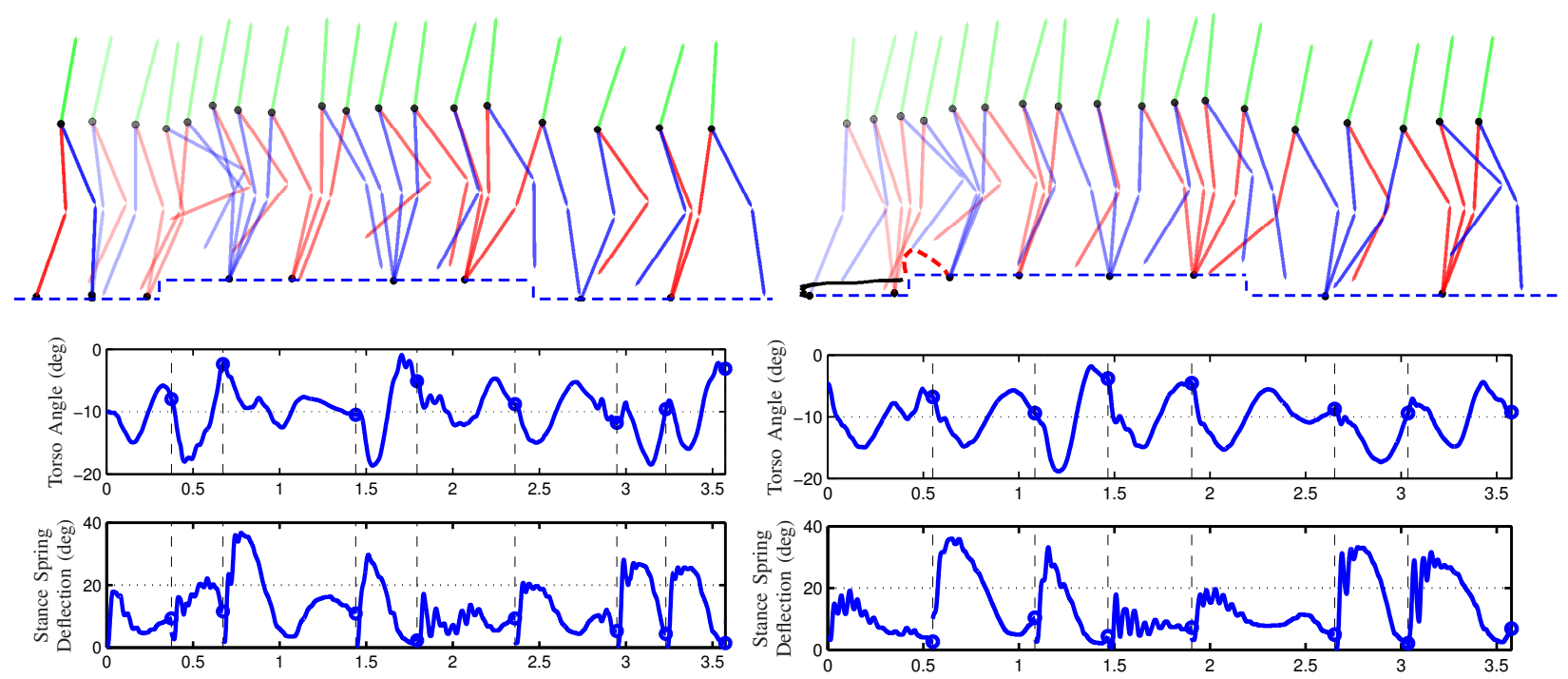

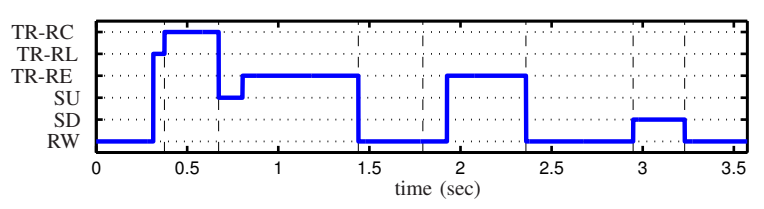

(a)

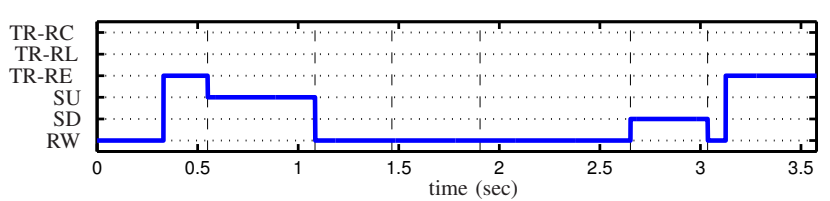

(b)

Fig. 13: Experimental data of step-up and step-down of $10.5 \mathrm{~cm}$ platform. In the stick figure illustration, black circle at the leg end indicates stance toe. Torso angle (top), $s\left(q_{\mathrm{s}}\right)$ (middle), and types of controllers (bottom) applied are shown next, respectively. In these figures, blue hollow circle and black dashed line indicate the end of the step. RW, SD, SU, TR-RE, TR-RL, TR-RC refer to walking on flat ground, step-down, step-up, rapid-elevation of the swing leg, rapid-lowering of the swing, and recovery, respectively. Black solid line and red dashed line in the stick figure illustration demonstrate the change in swing leg path due to the activation of the tripping-reflex controller. A detailed illustration of the controller transition and swing leg modification during stepping-up are shown in Figure 14. (a) First lap (b) Second lap.

On the other hand, on the second lap, the rapid-elevation controller is triggered in response to the early perturbation as shown in Figure 14(b). With either controller, MABEL could successfully step upon a $10.5 \mathrm{~cm}$ platform. We verified that MABEL could ascend a platform with a height of up to $12.5 \mathrm{~cm}$, which is $12.5 \%$ of the leg length. In response to step-down disturbances of the $10.5 \mathrm{~cm}$ height, the stepdown controller is activated as shown in the bottom figures of Figure 14(a) and (b), and step-down disturbances were accommodated by the controller.

\section{Consecutive Two Step-ups and One Large Step-down of $18.5 \mathrm{~cm}$ platform}

For the next experiment, we built a platform with two stair-steps, one with a height of $10.5 \mathrm{~cm}$ and the other with a height of $8 \mathrm{~cm}$ as shown in Figure 15. MABEL walked up two consecutive risers, took two steps of level walking on top of the platform, and stepped off from the platform with one step $(18.5 \mathrm{~cm}$ step-down, which is $18.5 \%$ of the leg length), while skipping a stair-step placed between the top of the platform and the ground. The bottom graph of Figure 15b shows the horizontal hip velocity. In the graph, the horizontal hip velocity at the end of the two stair ascents is $0.9 \mathrm{~m} / \mathrm{sec}$ (see the blue circle at $1.295 \mathrm{sec}$ in the hip velocity graphs.) which is negligibly different from $0.93 \mathrm{~m} / \mathrm{sec}$ of the average flat-walking hip velocity measured at the end of the step. After step-down from the $18.5 \mathrm{~cm}$ high platform, hip velocity at the end of step was increased to $1.8 \mathrm{~m} / \mathrm{sec}$ (see the blue circle at $3.148 \mathrm{sec}$ ), but reduced to $1.12 \mathrm{~m} / \mathrm{sec}$ rapidly at the ensuing step. From these analyses of the hip velocity, it is observed that hip velocity is successfully regulated by the proposed controller in response to large step-up and step-down disturbances. The rapid-elevation controller in the middle of the step-down phase is activated from $2.919 \mathrm{sec}$ to $3.148 \mathrm{sec}$ because the swing foot struck a stair between the top of the platform and the ground during a step-down event. Figure 16 shows control torques. Torques are saturated at $10 \mathrm{Nm}$ for Motor Leg Angle and $20 \mathrm{Nm}$ for Motor Leg Shape.

A similar experiment was conducted on the same platform, but this time MABEL took two steps during the step-down as shown in Figure 17. Therefore, the step-down controller was executed twice in succession.

\section{One Step-up, One Regular-Walking Step, and One Step- down of $10.5 \mathrm{~cm}$ Platform}

In this experiment, MABEL stepped upon a $10.5 \mathrm{~cm}$ platform, took one step on the top of the platform, and stepped off the platform as shown in Figure 18. Figure 19 shows a stick figure illustration of the step-down phase in Figure 19a and the ensuing step in Figure 19b, along with torso angle, spring deflection, and the types of controllers applied during the experiment in Figure 19c. As shown in Figure 19, tripping over 

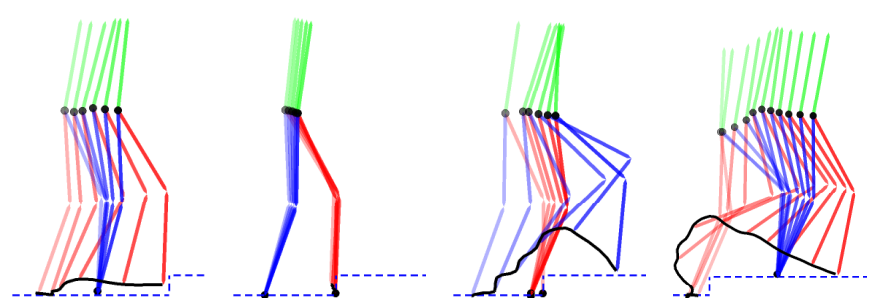

(a)
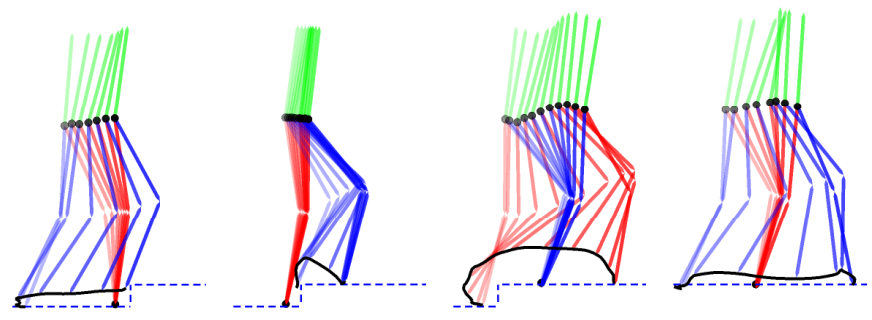

(b)

Fig. 14: Detailed illustration of the controller transition and swing leg modification during stepping-up of Experiment B. Black circle at leg end indicates stance toe. (a) On the first lap, the rapid-lowering controller is activated in response to late tripping. First two figures show rapid-lowering of the swing leg, and last two figures display the action of the recovery controller. In part (b), on the second lap, the rapid-elevation controller is triggered in response to early tripping. Rapidelevation of the swing leg occurs in the second figure, and at the ensuing step (last two figures), the step-up controller is activated.

the ground occurred at the end of the step. In response to the tripping event, the rapid-elevation controller was applied (see 0.411 sec of Figure 19c), but the swing foot could not clear the ground. Therefore, the recovery controller was triggered at the ensuing step instead of the regular-walking controller. This clearly shows use of the transition from rapid-elevation phase to recovery phase as shown in Figure 5.

\section{E. Discussion of the Experiments}

Although MABEL was able to accommodate various combinations of terrain as explained above, several tests conducted on certain combinations of disturbances showed limitations in the finite-state machine. For example, MABEL consistently failed to accommodate a platform with consecutive stepup and step-down disturbances with a height greater than $10.5 \mathrm{~cm}$ although experiments on this type of ground variations were repeated several times. Similar results were obtained by other experiments on a platform with consecutive step-down and step-up disturbances with change in height greater than $10.5 \mathrm{~cm}$. Typical platforms are shown in Figure 20. As stated previously, for shorter platforms, the robot could accommodate such combinations of disturbances.

It can be conjectured that the reason for these failures is that the robot's state after the large step-up or step-down disturbance is not in the "basin of attraction" of the ensuing step-down or step-up controller. Quantifying these basins of
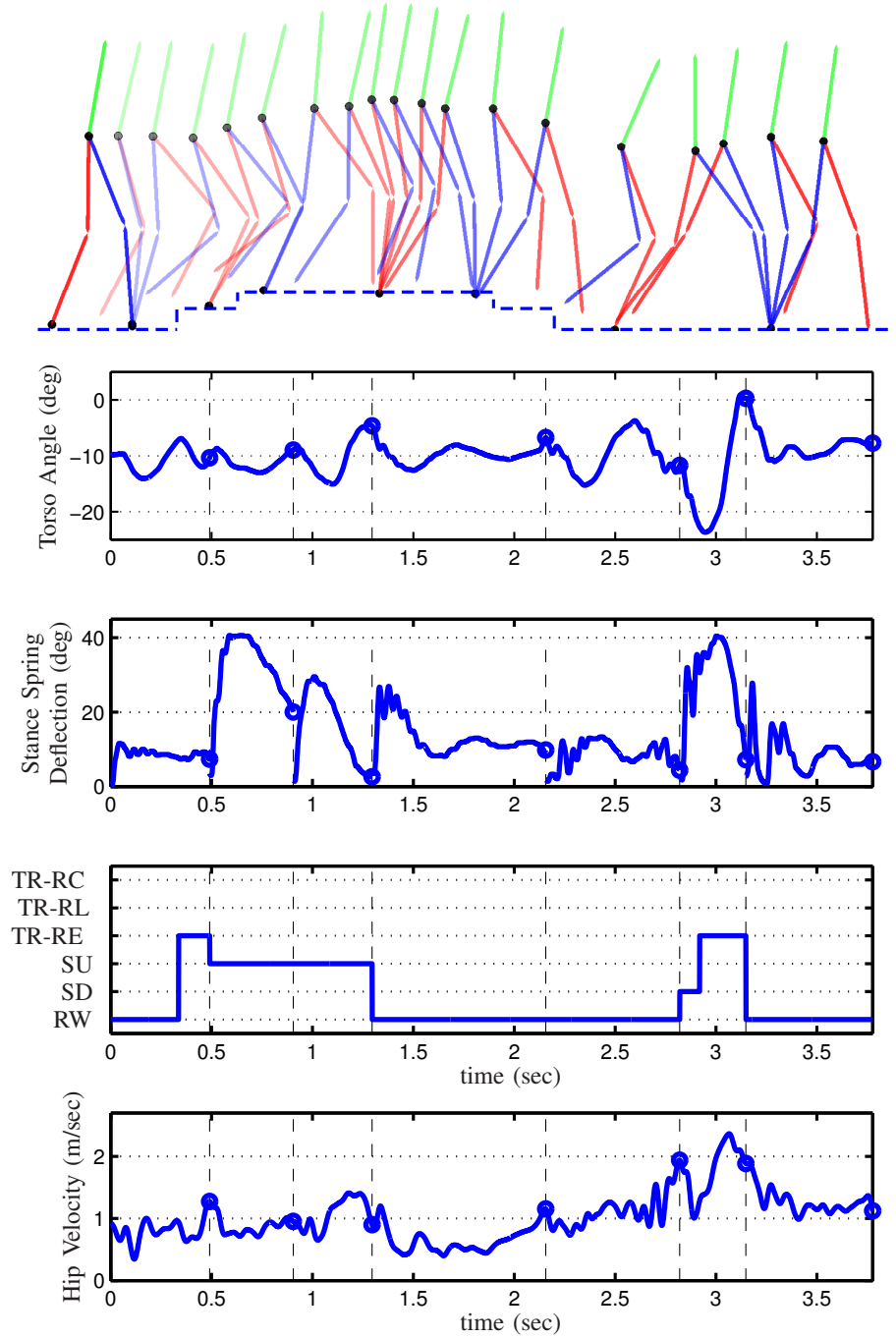

Fig. 15: Experimental data of consecutive two step-up and one large step-down of $18.5 \mathrm{~cm}$ platform. (a) Stick figure illustration. Black circle at the toe indicates stance toe. (b) Torso angle, spring deflection, types of controllers applied, and horizontal hip velocity are shown, respectively. Blue hollow circle and black dashed line display end of the step.
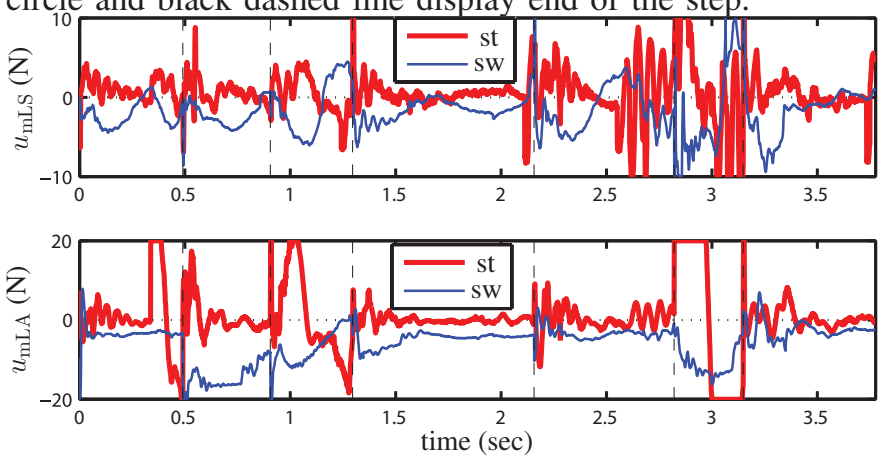

Fig. 16: Experimental data of consecutive two step-up and one large step-down of $18.5 \mathrm{~cm}$ platform. Torques are shown.

attraction and redesigning the step-up or step-down controllers are extremely challenging tasks.

\section{CONCLUSION}

A finite-state machine has been designed to handle various kinds of disturbances including blind step-downs and step- 


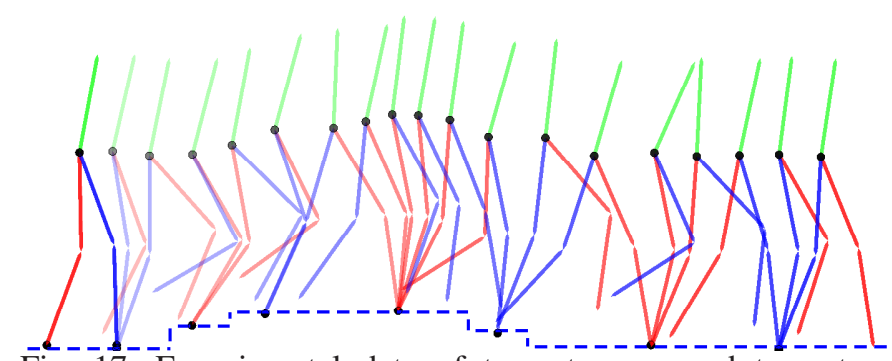

Fig. 17: Experimental data of two step-ups and two stepdowns. Black circle at the toe indicates stance toe.

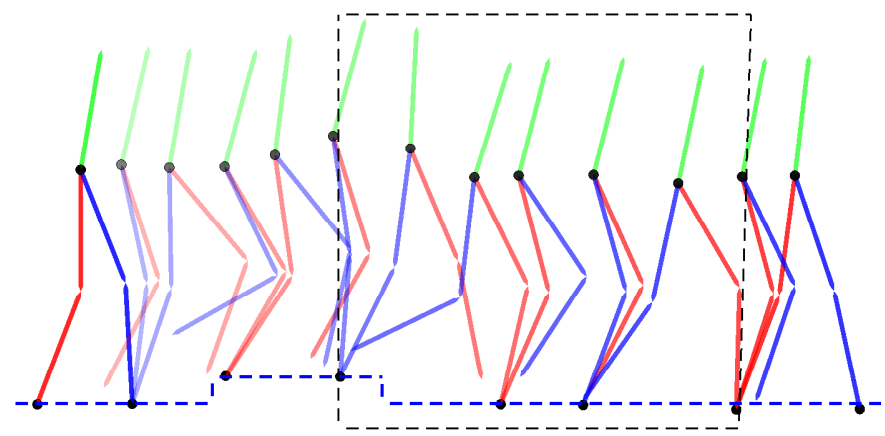

Fig. 18: Experimental data of one step-up, one regular-walking step, and one step-down of $10.5 \mathrm{~cm}$ platform. Steps in black dashed square is redisplayed in Figure 19.

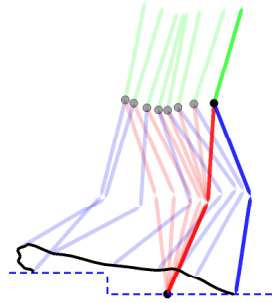

(a)
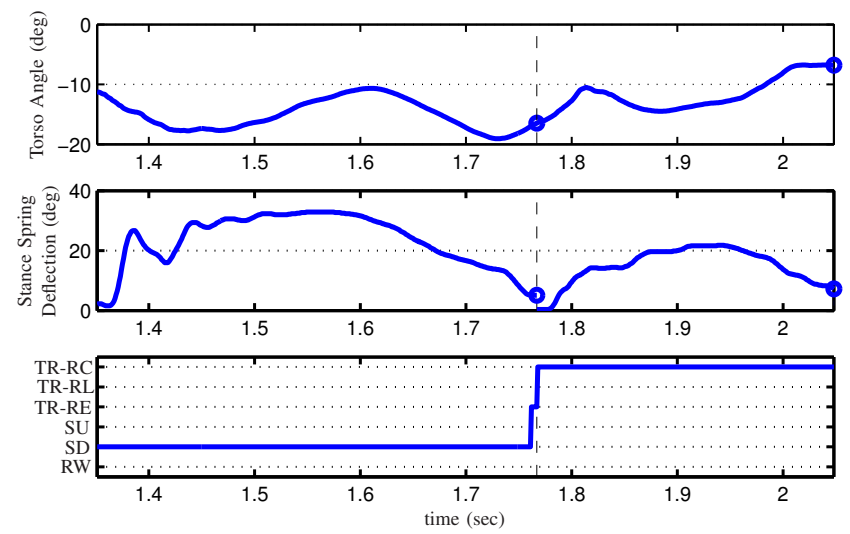

(c)

Fig. 19: Experimental data of one step-up, one regular-walking step, and one step-down of $10.5 \mathrm{~cm}$ platform. In this figure, only step-down and ensuing step are shown. Stick figure illustration of (a) step-down, and (b) ensuing step. Black circle at the toe and black solid line indicate stance toe and a trajectory of swing toe, respectively. Premature impact with the ground is observed during the step-down. (c) Torso angle, spring deflection, and types of controllers used in the experiments. Blue hollow circle and vertical dashed black line illustrate at the end of the step.
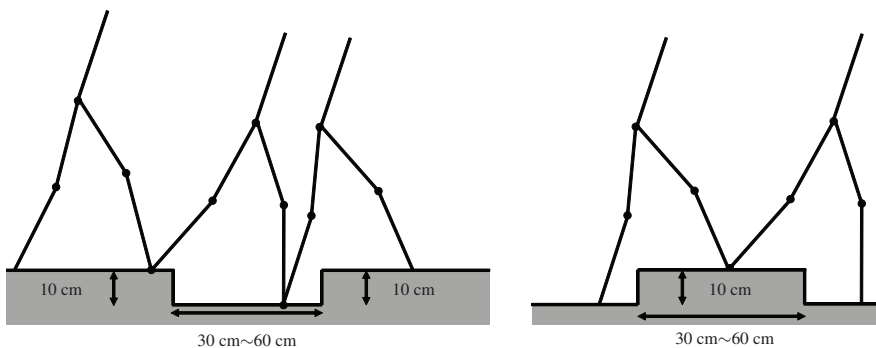

Fig. 20: Typical examples of terrain causing the robot to fall. On the other hand, if the robot had at least one regular-walking step between such obstacles, it did not fall; see, for example, Figures 15, 17, and 18.

ups of considerable height, and tripping over obstacles. The proposed finite-state machine consists of a regular-walking controller for walking on flat ground and three special-purpose controllers, namely step-down, step-up, and tripping-reflex controllers, whose objective is the safe continuation of walking in response to a large disturbance in ground height or tripping over obstacles.

Each of the controllers employs active force control at the stance knee, with the feature of being able to easily vary its stiffness and damping. When optimization was used to tune the stance-knee stiffness of the regular-walking, step-down, and step-up controllers, a pattern similar to that observed in humans was found: a virtual compliant element for the step-down controller is stiffer than for the regular walking controller, and a softer virtual compliant element was found for the step-up controller.

The tripping-reflex controller is designed to behave in different manners with respect to early or late tripping: a rapid elevating strategy of the swing leg is activated in response to early tripping while a rapid lowering strategy is used in response to late tripping. This rapid lowering strategy is followed by a recovery phase which is designed to reject the previous step's disturbance.

Experimentation with this controller showed MABEL traversing various kinds of obstacles including $12.5 \mathrm{~cm}$ blind step-ups, and $18.5 \mathrm{~cm}$ blind step-downs, as well as various combinations of step-ups and step-downs, such as short stacks of stairs.

Although the proposed finite-state machine shows good performance on various terrain profiles, the video in [39] shows limited controller performance to attenuate the influence of random variations in ground height on the robot's speed. To improve controller performance in the face of such disturbances, it would be interesting to explore an outer-loop discrete-event-based control design which can be layered onto the present controller.

\section{REFERENCES}

[1] K. Byl and R. Tedrake, "Metastable walking machines," International Journal of Robotics Research, vol. 28, pp. 1040-1064, August 2009.

[2] R. T. Katie Byl, "Metastable walking on stochastically rough terrain," in Proceedings of Robotics: Science and Systems IV, Zurich, Switzerland, June 2008.

[3] K. Byl and R. Tedrake, "Approximate optimal control of the compass gait on rough terrain," in IEEE International Conference on Robotics and Automation, may 2008, pp. $1258-1263$. 
[4] D. Hobbelen and M. Wisse, "A disturbance rejection measure for limit cycle walkers: The gait sensitivity norm," IEEE Transactions on Robotics, vol. 23, no. 6, pp. 1213 -1224, dec. 2007.

[5] — "Swing-leg retraction for limit cycle walkers improves disturbance rejection," IEEE Transactions on Robotics, vol. 24, no. 2, pp. 377 -389, april 2008.

[6] M. Wisse, A. L. Schwab, R. Q. van der Linde, and F. C. T. van der Helm, "How to keep from falling forward: Elementary swing leg action for passive dynamic walkers," IEEE Transactions on Robotics, vol. 21, no. 3, pp. 393-401, June 2005.

[7] I. R. Manchester, U. Mettin, F. Iida, and R. Tedrake, "Stable dynamic walking over uneven terrain," The International Journal of Robotics Research, vol. 30, no. 3, pp. 265-279, 2011.

[8] I. R. Manchester, "Transverse dynamics and regions of stability for nonlinear hybrid limit cycles." in Proceedings of the 18th IFAC World Congress, 2011.

[9] C. Sabourin, O. Bruneau, and G. Buche, "Control strategy for the robust dynamic walk of a biped robot," The International Journal of Robotics Research, vol. 25, no. 9, pp. 843-860, Sept. 2006.

[10] Q. Huang and Y. Nakamura, "Sensory reflex control for humanoid walking," IEEE Transactions on Robotics, vol. 21, no. 5, pp. 977 984, oct. 2005.

[11] G. N. Boone and J. K. Hodgins, "Slipping and tripping reflexes for bipedal robots," Autonomous Robots, vol. 4, pp. 259-271, July 1997.

[12] J. Pratt, C.-M. Chew, A. Torres, P. Dilworth, and G. Pratt, "Virtual model control: An intuitive approach for bipedal locomotion," The International Journal of Robotics Research, vol. 20, no. 2, pp. 129-143, 2001.

[13] J. Grizzle, J. Hurst, B. Morris, H.-W. Park, and K. Sreenath, "MABEL, a new robotic bipedal walker and runner," in American Control Conference, St. Louis, MO, USA, June 2009, pp. 2030-2036.

[14] K. Sreenath, H.-W. Park, I. Poulakakis, and J. W. Grizzle, "A compliant hybrid zero dynamics controller for stable, efficient and fast bipedal walking on MABEL," The International Journal of Robotics Research, vol. 30, no. 9, pp. 1170-1193, Aug 2011.

[15] H.-W. Park, K. Sreenath, J. Hurst, and J. Grizzle, "Identification of a bipedal robot with a compliant drivetrain," IEEE Control Systems Magazine, vol. 31, no. 2, pp. 63 -88, april 2011.

[16] J. W. Hurst, "The role and implementation of compliance in legged locomotion," Ph.D. dissertation, Robotics Institute, Carnegie Mellon University, Pittsburgh, PA, August 2008.

[17] R. Alexander, "Three uses for springs in legged locomotion," The International Journal of Robotics Research, vol. 9, no. 2, pp. 53-61, 1990.

[18] W. Khalil and E. Dombre, Modeling, Identification and Control of Robots. Bristol, PA, USA: Taylor \& Francis, Inc., 2002.

[19] Y. Hurmuzlu and D. B. Marghitu, "Rigid Body Collisions of Planar Kinematic Chains With Multiple Contact Points," The International Journal of Robotics Research, vol. 13, no. 1, pp. 82-92, 1994.

[20] C. Canudas de Wit, H. Olsson, K. Astrom, and P. Lischinsky, "A new model for control of systems with friction," IEEE Transactions on Automatic Control, vol. 40, no. 3, pp. 419-425, Mar. 1995.

[21] F. Plestan, J. Grizzle, E. Westervelt, and G. Abba, "Stable walking of a 7-DOF biped robot," IEEE Transactions on Robotics and Automation, vol. 19, no. 4, pp. 653-668, Aug. 2003.

[22] E. R. Westervelt, J. W. Grizzle, C. Chevallereau, J. H. Choi, and B. Morris, Feedback Control of Dynamic Bipedal Robot Locomotion. Taylor \& Francis/CRC Press, 2007.

[23] C. Canudas de Wit, "On the concept of virtual constraints as a tool for walking robot control and balancing," Annual Reviews in Control, vol. 28, no. 2, pp. 157-166, 2004.

[24] H.-W. Park. (2010) First attempt at walking over rough ground for bipedal robot mabel. Youtube Video. [Online]. Available: http://youtu.be/IlWIWf4daNs

[25] T. Yang, E. Westervelt, and A. Serrani, "A framework for the control of stable aperiodic walking in underactuated planar bipeds," in IEEE International Conference on Robotics and Automation, Roma, Italy, April 2007, pp. 4661-4666.

[26] K. Sreenath, H.-W. Park, , and J. W. Grizzle, "Embedding active force control within the compliant hybrid zero dynamics to achieve stable, fast running on mabel," The International Journal of Robotics Research, 2012, submitted.

[27] K. Sreenath, "Feedback control of a bipedal walker and runner with compliance," Ph.D. dissertation, The University of Michigan, 2011.

[28] H.-W. Park, K. Sreenath, A. Ramezani, and J. Grizzle, "Switching control design for accommodating large step-down disturbances in bipedal robot walking," in IEEE International Conference on Robotics and Automation, Saint Paul, USA, May 2012, pp. 45-50.
[29] S. D. Prentice, E. N. Hasler, J. J. Groves, and J. S. Frank, "Locomotor adaptations for changes in the slope of the walking surface," Gait \& Posture, vol. 20, no. 3, pp. 255 - 265, 2004.

[30] K. Kawamura, A. Tokuhiro, and H. Takechi, "Gait analysis of slope walking: a study on step length, stride width, time factors and deviation in the center of pressure," Acta Med Okayama, vol. 45, no. 3, pp. 179 $-184,1991$.

[31] A. Tokuhiro, H. Nagashima, and H. Takechi, "Electromyographic kinesiology of lower extremity muscles during slope walking," Arch Phys Med Rehabil, vol. 66, no. 9, pp. 610 - 613, 1985.

[32] S. Grimmer, M. Ernst, M. Günther, and R. Blickhan, "Running on uneven ground: leg adjustment to vertical steps and self-stability," Journal of Experimental Biology, vol. 211, no. 18, pp. 2989-3000, 2008.

[33] R. Müller and R. Blickhan, "Running on uneven ground: Leg adjustments to altered ground level," Human Movement Science, vol. 29, no. 4, pp. $578-589,2010$.

[34] J. J. Eng, D. A. Winter, and A. E. Patla, "Strategies for recovery from a trip in early and late swing during human walking," Experimental Brain Research, vol. 102, pp. 339-349, 1994.

[35] —, "Intralimb dynamics simplify reactive control strategies during locomotion," Journal of Biomechanics, vol. 30, no. 6, pp. 581 - 588, 1997.

[36] R. W. Sinnet, M. J. Powell, R. P. Shah, and A. D. Ames, "A humaninspired hybrid control approach to bipedal robotic walking," in 18th IFAC World Congress, 2011.

[37] H.-W. Park, "Control of a bipedal robot walker on rough terrain," Ph.D. dissertation, The University of Michigan, 2012.

[38] —. (2012) Bipedal robot mabel traverses uneven terrain using touch instead of sight. Youtube Video. [Online]. Available: http: //youtu.be/MhPSZOUgZ_8

[39] —. (2011) Bipedal robot mabel walks over randomly varying ground: Experiment no. 1. Youtube Video. [Online]. Available: http://youtu.be/m8-j7s6-bzE

\section{APPENDIX A \\ MODIFICATION ON ViRTUAL COMPLIANCE AND Calculation of EFFective STIFFness}

MABEL has three sources of compliance: physical springs installed on the torso, virtual compliance created by the leg shape motor, and compliance due to the cable stretch. Because the cable stretch is not included in the simplified design model, the stiffness of virtual compliance $k^{*}$ designed from optimization on simplified-model needs to be modified to take into account the effect of the cable stretch. This modification is based on the fact that the cable stretch and the virtual compliance form a series connection, and consequently, we can multiply $k^{*}$ by some correction factor $\gamma_{\mathrm{vc}}$ so that the series connection realizes the desired compliance $k^{*}$, namely,

$$
\frac{1}{k^{*}}=\frac{1}{\gamma_{\mathrm{vc}} k^{*}}+\frac{1}{k_{\text {cable }}},
$$

where $k_{\text {cable }}$ is the spring constant of the cable stretch seen at the motor leg shape angle. For the designed stiffness value of the regular-walking controller $k^{*, \mathrm{RW}}=1.8, \gamma_{\mathrm{vc}}=2.5$ is obtained from (23), and we use this correction factor for the step-up, step-down, and recovery controllers as well as to make the controller structure simpler.

Similarly, effective stiffness $k_{\text {eff }}$ which is the series summation of three sources of compliance can be calculated as follows:

$$
\frac{1}{k_{\mathrm{eff}}}=\frac{1}{k_{\mathrm{Bsp}}}+\frac{1}{\gamma_{\mathrm{vc}} k^{*}}+\frac{1}{k_{\mathrm{cable}}},
$$

where $k_{\mathrm{Bsp}}$ is the spring constant of physical spring, seen at the motor leg shape angle. 